\title{
$\mathrm{M}|\mathrm{R}| \mathrm{S}$ Internet Journal Nitride Semiconductor Research
}

\section{High-Speed Visible-Blind Resonant Cavity Enhanced AlGaN Schottky Photodiodes}

\author{
Necmi Biyikli ${ }^{1}$, Tolga Kartaloglu ${ }^{1}$, Orhan Aytur ${ }^{1}$, Ibrahim Kimukin ${ }^{2}$ and Ekmel Ozbay ${ }^{2}$ \\ ${ }^{1}$ Bilkent University Dept. of Electrical and Electronics Engineering, \\ ${ }^{2}$ Bilkent University Dept. of Physics,
}

(Received Friday, September 19, 2003; accepted Thursday, October 30, 2003)

We have designed, fabricated and tested resonant cavity enhanced visible-blind AlGaN-based Schottky photodiodes. The bottom mirror of the resonant cavity was formed with a 20 pair AlN/ $\mathrm{Al}_{0.2} \mathrm{Ga}_{0.8} \mathrm{~N}$ Bragg mirror. The devices were fabricated using a microwave compatible fabrication process. Au and indium-tin-oxide (ITO) thin films were used for Schottky contact formation. ITO and Au-Schottky devices exhibited resonant peaks with $0.153 \mathrm{~A} / \mathrm{W}$ and $0.046 \mathrm{~A} / \mathrm{W}$ responsivity values at $337 \mathrm{~nm}$ and $350 \mathrm{~nm}$ respectively. Temporal high-speed measurements at $357 \mathrm{~nm}$ resulted in fast pulse responses with pulse widths as short as $77 \mathrm{ps}$. The fastest UV detector had a 3-dB bandwidth of $780 \mathrm{MHz}$.

\section{Introduction}

Solar/visible-blind detection is vital for a number of critical applications including missile plume detection, flame/engine monitoring, chemical/biological agent sensing, and covert space-to-space communications [1]. With its wide bandgap and intrinsic visible-blind absorption spectrum, $\mathrm{Al}_{\mathrm{x}} \mathrm{Ga}_{1-\mathrm{x}} \mathrm{N}$ ternary alloy is the most promising material system for high-performance solar/visible-blind photodetectors [2]. High-performance ultraviolet (UV) detectors were reported using AlGaN-based Schottky [3] [4] [5] [6], MSM [7] [8] [9], pin [10], [11], and avalanche type of photodiodes (PDs) [12]. These devices consisted of conventional, single-pass detector structures. On the other hand, resonant cavity enhanced (RCE) detector structure offers selective enhancement of device responsivity [13]. The RCE-PD exhibits a multi-pass detection scheme at the cavity resonance wavelengths, which leads to improved efficiency performance.

High performance infrared RCE-PDs using AlGaAs and InGaAs material systems were previously reported [14] [15] [16]. Recently, Kishino et al. have demonstrated the first UV RCE-PD with an AlGaN-based MSM PD, where they have reported resonant peaks around $360 \mathrm{~nm}$ [17]. In this paper, we report on the design, fabrication, and characterization of high-speed RCE AlGaN Schottky PDs. Au and ITO-Schottky devices were fabricated and compared in terms of detector performance. A peak responsivity of $0.153 \mathrm{~A} / \mathrm{W}$ at $337 \mathrm{~nm}$ and a $3-\mathrm{dB}$ bandwidth of $780 \mathrm{MHz}$ were obtained with ITO and Au-Schottky RCE-PDs respectively.

\section{Experimental}

\subsection{Epitaxial Design}

For the design of the RCE-PD structure, a transfermatrix-method (TMM) based simulation program was used. The detector was designed to achieve a resonant enhancement around $350 \mathrm{~nm}$. The spectral complex refractive index data of $\mathrm{Al}_{\mathrm{x}} \mathrm{Ga}_{1-\mathrm{x}} \mathrm{N}$ needed for the simulations were obtained from Ref. 18. Figure 1 shows the epitaxial layer structure of the designed RCE-PD wafer. A lightly doped $\left(\mathrm{n}=5 \times 10^{16} \mathrm{~cm}^{-3}\right) 70 \mathrm{~nm}$ thick GaN absorption layer was used as the detector active region. All other detector layers consisted of $\mathrm{Al}_{\mathrm{x}} \mathrm{Ga}_{1-\mathrm{x}} \mathrm{N}$ layers with $\mathrm{Al}$ mole fraction larger than $20 \%$. The bottom mirror of the resonant cavity was formed by a 20 pair AlN/ $\mathrm{Al}_{0.2} \mathrm{Ga}_{0.8} \mathrm{~N}$ DBR. The Bragg mirror was centered at the operation wavelength of $\lambda=360 \mathrm{~nm}$. The $\lambda / 4$ layer thicknesses required for a highly reflecting $\mathrm{AlN} / \mathrm{Al}_{0.2} \mathrm{Ga}_{0.8} \mathrm{~N}$ quarter wave stack at this wavelength were determined as $43.3 \mathrm{~nm}$ and $36.7 \mathrm{~nm}$ respectively. Since the cut-off wavelength of $\mathrm{Al}_{0.2} \mathrm{Ga}_{0.8} \mathrm{~N}$ is around $330 \mathrm{~nm}$, all cavity 
layers except the GaN absorption layer were transparent for $\lambda \geq 330 \mathrm{~nm}$. The $300 \mathrm{~nm}$ thick, highly doped $\left(\mathrm{n}=2 \times 10^{18} \mathrm{~cm}^{-3}\right) \mathrm{Al}_{0.2} \mathrm{Ga}_{0.8} \mathrm{~N}$ layer was designed as the ohmic contact layer. To electrically isolate the device mesas, a $200 \mathrm{~nm}$ thick undoped $\mathrm{Al}_{0.2} \mathrm{Ga}_{0.8} \mathrm{~N}$ layer was grown above the bottom DBR.

\subsection{Device Fabrication}

The AlGaN/GaN epitaxial layers were grown on a sapphire substrate using MOCVD. The devices were fabricated using a microwave compatible fabrication process consisting of five mask levels: ohmic contact formation, mesa isolation, Schottky contact formation, surface passivation, and metalization of interconnect pads. First, the ohmic contacts were defined via reactive ion etching (RIE) under $\mathrm{CCl}_{2} \mathrm{~F}_{2}$ plasma, $20 \mathrm{sccm}$ gas flow-rate and $100 \mathrm{~W}$ RF power. A controlled etch was done to stop within the $\mathrm{n}+\mathrm{Al}_{0.2} \mathrm{Ga}_{0.8} \mathrm{~N}$ layer. The final etch depth was $\sim 0.35 \mu \mathrm{m}$. Afterwards Ti/Al contacts were deposited using thermal evaporation and standard lift-off process. The contacts were annealed at $600{ }^{\circ} \mathrm{C}$ for 60 seconds in a rapid thermal annealing (RTA) system. Mesa structures of the devices were formed via the same RIE process, by etching all the layers down to the undoped mesa isolation layer. Third step was the deposition of the Schottky contact layer. Au and ITO were used for making Schottky contacts to GaN. $\sim 100 \mathrm{~nm}$ thick ITO and $\sim 10 \mathrm{~nm}$ thick Au films were RF-sputtered and thermally evaporated on different samples respectively. The ITO films were etched to define the contact regions using dilute $\mathrm{HF}: \mathrm{H}_{2} \mathrm{O}$ solution, whereas the $\mathrm{Au}$ films were lifted-off in acetone solution. Next, to passivate the sample surface and protect the Schottky contacts, a $\sim 120 \mathrm{~nm}$ thick $\mathrm{Si}_{3} \mathrm{~N}_{4}$ film was deposited onto the samples by plasma-enhanced-chemical-vapor-deposition (PECVD). Finally, a $~ 0.7 \mu \mathrm{m}$ thick Ti/Au interconnect metal was deposited and lifted-off to connect the Schottky layers to coplanar waveguide transmission line pads.

\subsection{Device Testing}

After device fabrication, current-voltage (I-V), spectral responsivity, and high-speed characterizations were carried out. Before device fabrication, reflectivity characterization was made and the results were compared with the simulation results to estimate the actual layer thicknesses. The spectral reflectivity measurements were done using a fiber-optic based setup. Ocean Optics PC2000-UV-VIS PC Plug-in Fiber Optic Spectrometer was used in this measurement, which operated in the 200-850 nm wavelength region. The I-V characterization was carried out using a Hewlett Packard HP4142B DC parametric measurement instrument, DC probes with triax output and low-noise triax cables. Spectral responsivity measurements were done using a $175 \mathrm{~W}$ xenon light-source, 1/4 m Digikrom DK240 monochromator, multi-mode UV fiber, DC voltage source, SR830 DSP lock-in amplifier and a Newport model 1830-C calibrated optical power-meter. Xenon lamp output was fed into the monochromator. The monochromator output was chopped and coupled to the fiber using a UVenhanced focusing lens. The detectors were illuminated by the optical output coming out from the fiber which was calibrated using a NIST-calibrated Si photodetector. The resulting photocurrent was measured using the lock-in amplifier. Temporal high-frequency measurements were done at $357 \mathrm{~nm}$. UV pulses with sub-picosecond pulsewidths were generated using second harmonic generation (SHG) process. A Coherent Mira 900F model femtosecond mode-locked Ti:sapphire laser was used to generate the pump beam at $714 \mathrm{~nm}$. The pump pulses were produced with $76 \mathrm{MHz}$ repetition rate and $140 \mathrm{fs}$ pulse duration. These pulses were frequency doubled to generate a second harmonic beam at $357 \mathrm{~nm}$ using a $0.5 \mathrm{~mm}$ thick type-I $\beta$-BaB2O4 (BBO) crystal. The resulting $357 \mathrm{~nm}$ pulses had pulsewidths below $1 \mathrm{ps}$ and were focused on-to the devices using UV-enhanced mirrors and lenses. To measure the pulse response of the detectors at $267 \mathrm{~nm}$, another BBO crystal was added to this setup. Using the pump beam at $800 \mathrm{~nm}, 267 \mathrm{~nm}$ pulses were generated by sum frequency generation process [18]. The detectors were biased using a DC voltage source and a $40 \mathrm{GHz}$ bias-tee. The resulting temporal pulse responses were observed on a $50 \mathrm{GHz}$ sampling oscilloscope.

\section{Results and Discussion}

In order to estimate the actual epitaxial layer thicknesses, spectral reflectivity was measured before device fabrication. Comparing the measured spectral reflectivity with the simulation results of the ideal detector structure, significant differences between the experimental and computational data were observed. Our original simulations predicted a highly reflective mirror between 340 and $380 \mathrm{~nm}$. However, the experimental reflectivity data showed that the long wavelength edge of the mirror had blue-shifted nearly $25 \mathrm{~nm}$ towards $355 \mathrm{~nm}$. This result convinced us that the layers were grown shorter than the original design. Figure 2 shows the reflectivity measurements along with the simulation results obtained with $\sim 10 \%$ shorter cavity and mirror layers. With this modified epitaxial layer structure, a reasonable agreement between the experimental and theoretical reflectivity was achieved. A blue-shifted peak reflectivity of $52 \%$ around $345 \mathrm{~nm}$ was measured.

The fabricated AlGaN RCE Schottky PDs exhibited low dark currents at small reverse bias values. Both $\mathrm{Au}$ 
and ITO-Schottky samples had dark currents below 10 $\mathrm{pA}$ at reverse bias of $1.5 \mathrm{~V}$. Typical breakdown voltages measured were around $10 \mathrm{~V}$, and ITO devices showed larger turn-on voltages than Au devices (Figure 3). The measured dark current density of a $100 \times 100 \mu \mathrm{m}^{2} \mathrm{RCE}$ ITO-Schottky PD is plotted in Figure $4.1 .3 \times 10^{-8} \mathrm{~A} / \mathrm{cm}^{2}$ dark current density was obtained at $1 \mathrm{~V}$ reverse bias.

The spectral photoresponse measurements of the AlGaN RCE-PDs were performed in the $320-420 \mathrm{~nm}$ spectral region. Figure 5 shows the measured spectral quantum efficiency of Au and ITO-Schottky RCE-PDs under different bias conditions. When no reverse bias voltage was applied, peak efficiencies of $20 \%$ and $7 \%$ were measured with ITO and Au samples respectively. Photoresponse of both samples increased with reverse bias. ITO-Schottky PDs exhibited a resonant peak of $56 \%$ at $337 \mathrm{~nm}$ under $1 \mathrm{~V}$ reverse bias. A weaker resonance was observed in Au-Schottky sample with a peak efficiency of $19 \%$ at $350 \mathrm{~nm}$ under $4 \mathrm{~V}$ reverse bias.

The difference in the peak responses for ITO and Au-Schottky devices can be explained by two factors: absorption in Schottky layer and matching of top mirror reflectivity. Absorption in Schottky layer directly decreases the achievable peak efficiency. Our simulations predicted that a $100 \AA$ thick Au film absorbed $\sim 35 \%$ of incident radiation around $350 \mathrm{~nm}$, whereas for a $100 \mathrm{~nm}$ thick ITO layer this loss was $\sim 15 \%$. Top mirror reflectivity plays an important role for optimum RCE operation. In an RCE-PD, enhancement is best observed when $R_{1}=R_{2} e^{-2 \alpha d}$ condition is satisfied, where $R_{1}, R_{2}$, and $\alpha$ corresponds to the top mirror reflectivity, bottom mirror reflectivity, and absorption coefficient of the active layer respectively. In our experiment, with $R_{2} \approx$ $52 \%$, maximum enhancement is achieved with $14 \%$ and $12 \%$ top mirror reflectivity at $350 \mathrm{~nm}$ and $337 \mathrm{~nm}$. ITO film displays a reflectivity of $\sim 16 \%$ which was close to the optimum value of $12 \%$. Reflectivity of Au film (34\% at $350 \mathrm{~nm}$ ), however, deviated significantly from the optimum condition, resulting in a weaker RCE effect. Figure 6 shows the measured spectral responsivity curves with peak values of $153 \mathrm{~mA} / \mathrm{W}$ and $46 \mathrm{~mA} / \mathrm{W}$ for biased ITO and Au-Schottky samples respectively. UV/ visible rejection performance of $\sim 1000$ and $\sim 600$ were measured for ITO and Au-Schottky PDs.

High-speed measurements were performed at two different wavelengths: $357 \mathrm{~nm}$ (near-resonance wavelength) and at $267 \mathrm{~nm}$. At both wavelengths, both RCE Schottky PD samples exhibited short rise times and longer decay times. We have measured and analyzed the temporal pulse responses as a function of applied reverse bias voltage, active device area, measurement wavelength, and Schottky contact material.
Figure 7 shows the bias dependence of high-speed responses obtained with Au-Schottky PDs measured at $357 \mathrm{~nm}$. Faster responses with higher peak amplitudes were measured with increasing reverse bias voltages. Under low bias voltages, some part of the undoped GaN/ $\mathrm{Al}_{0.2} \mathrm{Ga}_{0.8} \mathrm{~N}$ layers were not depleted, resulting in a diffusion current component. This diffusion component is observed as a slowly decaying tail in the pulse response. As the undoped layers were totally depleted under higher reverse bias voltages, carrier diffusion was eliminated and all the photo-generated carriers were swept towards the contact layers, resulting in faster pulse responses with shorter decaying tails. For a $150 \times 150$ $\mu \mathrm{m}^{2}$ device, full-width-at-half-maximum (FWHM) decreased from $870 \mathrm{ps}$ to $314 \mathrm{ps}$ as applied reverse bias increased from $2 \mathrm{~V}$ to $15 \mathrm{~V}$.

The area dependence of the measured high-speed performance of the Schottky PDs was in parallel to our expectations. Theoretically, better high-speed performance should be achieved with smaller device areas, due to smaller capacitance, and therefore smaller RC time constants. This was the case observed in our measurements. For Au-Schottky PDs, as the device diameter was changed from $200 \mu \mathrm{m}$ to $30 \mu \mathrm{m}$, FWHM decreased from 333 ps to $77 \mathrm{ps}$ at $15 \mathrm{~V}$ reverse bias. A similar behavior was observed for ITO-Schottky PDs, which exhibited slower responses with a minimum FWHM of $154 \mathrm{ps}$ at $357 \mathrm{~nm}$. Figure 8(a) and (b) show the area dependence of the $357 \mathrm{~nm}$ pulse response for $\mathrm{Au}$ and ITO-Schottky devices respectively.

The high-speed measurements at $267 \mathrm{~nm}$ resulted in slower pulse responses. This was an expected result, since the $\mathrm{Al}_{0.2} \mathrm{Ga}_{0.8} \mathrm{~N}$ cavity layers were not transparent at this wavelength. In this case, additional drift and diffusion currents are generated within the $\mathrm{Al}_{0.2} \mathrm{Ga}_{0.8} \mathrm{~N}$ cavity layers, resulting in longer response times. Figure 9(a) and (b) show the measured pulse responses under $267 \mathrm{~nm}$ illumination as a function of detector area. The fastest pulse-response obtained at this wavelength had pulse-widths of 110 ps and 234 ps for Au and ITO samples respectively. Figure 10(a) and (b) compares the pulse-widths obtained from $\mathrm{Au}$ and ITO RCE-PDs respectively measured at $357 \mathrm{~nm}$ and $267 \mathrm{~nm}$.

Comparing the measurement results obtained with $\mathrm{Au}$ and ITO-Schottky PDs, Au-Schottky PD samples exhibited better high-speed performance. To make a comparative analysis, the fastest pulse responses obtained from $30 \mu \mathrm{m} \mathrm{Au}$ and ITO-Schottky devices are normalized and plotted in Figure 11. The rise (charging) portion of the response for Au and ITO samples are very similar, both having short rise times. However, the decay (discharging) parts are drastically different. ITO-Schottky response shows a slower decay when compared to 
Au-Schottky response. Such a longer fall time indicates a higher RC time constant for ITO devices. Since the capacitance is the same for both devices, the difference in the response should originate from the total device resistance. ITO is known as transparent conductor, however when compared with Au, ITO is a weaker conductor with higher resistivity [15] [19]. This leads to a significantly higher series resistance in the device. It is this additional series resistance that increases the RC time constant of ITO-Schottky samples, resulting in longer decay times and slower pulse responses. The corresponding frequency responses are calculated using fast-Fourier transform (FFT) (see inset figure). 3-dB bandwidths of $150 \mathrm{MHz}$ and $780 \mathrm{MHz}$ were achieved for ITO and Au-Schottky PDs respectively. These results are the first high-speed results reported for visible-blind AlGaN RCE-PDs.

\section{Conclusions}

In summary, we have demonstrated high-speed, visibleblind RCE Schottky PDs on AlGaN epitaxial layers. AlGaN RCE-PDs with Au and ITO Schottky layers were fabricated. In spite of the lower-than-expected bottom $\mathrm{Al}_{0.2} \mathrm{Ga}_{0.8} \mathrm{~N} / \mathrm{AlN}$ Bragg mirror reflectivity, RCE effect was observed in both samples. ITO-Schottky samples displayed higher resonant peaks with a maximum peak responsivity of $0.153 \mathrm{~A} / \mathrm{W}$ at $337 \mathrm{~nm}$. Au-Schottky samples exhibited faster pulse responses with a minimum pulsewidth of $77 \mathrm{ps}$ at $357 \mathrm{~nm}$, and a corresponding 3-dB bandwidth of $780 \mathrm{MHz}$.

\section{ACKNOWLEDGMENTS}

This work was supported by NATO Grant No. SfP971970, Turkish Department of Defense Grant No. KOBRA-002, and FUSAM-03.

\section{REFERENCES}

[1] J. C. Carrano, T. Li, P. A. Grudowski, R. D. Dupuis, J. C. Campbell, IEEE Circuits \& Devices Mag. 15, 15 (1999).

[2] M. Razeghi, A. Rogalski, J. Appl. Phys. 79, 7433-7473 (1996).

[3] E. Monroy, F. Calle, J. L. Pau, F. J. Sanchez, E. Munoz, F. Omnes, B. Beaumont, P. Gibart, J. Appl. Phys. 88, 2081 (2000).

[4] S. L. Rumyantsev, N. Pala, M. S. Shur, R. Gaska, M. E. Levinshtein, V. Adivarahan, J. Yang, G. Simin, M. Asif Khan, Appl. Phys. Lett. 79, 866 (2001).

[5] N. Biyikli, T. Kartaloglu, O. Aytur, I. Kimukin, E. Ozbay, Appl. Phys. Lett. 79, 2838 (2001).

[6] N. Biyikli, O. Aytur, I. Kimukin, T. Tut, E. Ozbay, Appl. Phys. Lett. 81, 3272 (2002).

[7] B. Yang, D. J. H. Lambert, T. Li, C. J. Collins, M. M. Wong, U. Chowdhury, R. D. Dupuis, J. C. Campbell, Electron. Lett. 36, 1866 (2000).
[8] C. H. Chen, S. J. Chang, Y. K. Su, G. C. Chi, J. Y. Chi, C. A. Chang, J. K. Sheu, J. F. Chen, IEEE Photonics Technol. Lett. 13, 848 (2001).

[9] Sangwoo Seo, K. K. Lee, Sangbeom Kang, S. Huang, William A. Doolittle, N. M. Jokerst, A. S. Brown, M. A. Brooke, IEEE Photonics Technol. Lett. 14, 185 (2002).

[10] E. L. Tarsa, P. Kozodoy, J. Ibbetson, B. P. Keller, Appl. Phys. Lett. 77, 316 (2000).

[11] C. J. Collins, U. Chowdhury, M. M. Wong, B. Yang, A. L. Beck, R. D. Dupuis, J. C. Campbell, Appl. Phys. Lett. 80, 3754 (2002).

[12] J. C. Carrano, D. J. H. Lambert, C. J. Eiting, C. J. Collins, T. Li, S. Wang, B. Yang, A. L. Beck, R. D. Dupuis, J. C. Campbell, Appl. Phys. Lett. 76, 924 (2000).

[13] M. S. Unlu, S. Strite, J. Appl. Phys. 78, 607 (1995).

[14] E. Ozbay, I. Kimukin, N. Biyikli, O. Aytur, M. Gokkavas, G. Ulu, M. S. Unlu, R. P. Mirin, K. A. Bertness, D. H. Christensen, Appl. Phys. Lett. 74, 1072 (1999).

[15] N. Biyikli, I. Kimukin, O. Aytur, M. Gokkavas, M. S. Unlu, E. Ozbay, IEEE Photonics Technol. Lett. 13, 705 (2001).

[16] I. Kimukin, N. Biyikli, B. Butun, O. Aytur, M. S. Unlu, E. Ozbay, IEEE Photonics Technol. Lett. 14, 366 (2002).

[17] K. Kishino, M. Yonemaru, A. Kikuchi, Toyoura, Phys. Stat. Sol. A 188, 321 (2001).

[18] Necmi Biyikli, Tolga Kartaloglu, Orhan Aytur, Ibrahim Kimukin, Ekmel Ozbay, MRS Internet J. Nitride Semicond. Res. 8, 2 (2003).

[19] W. A. Wohlmuth, J. W. Seo, P. Fay, C. Caneau, I. Adesida, IEEE Photonics Technol. Lett. 9, 1388 (1997).

\section{FIGURES}

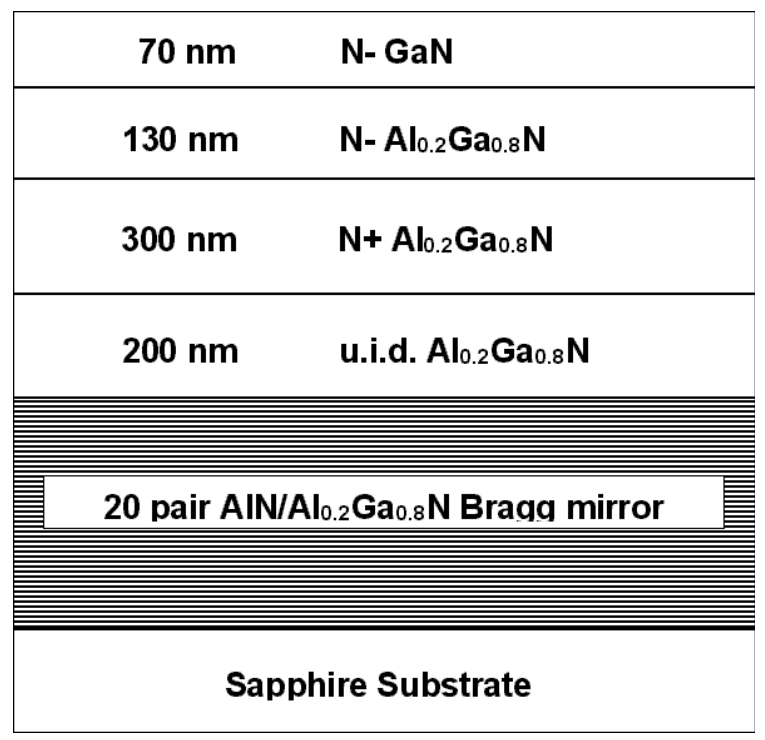

Figure 1. Epitaxial layer structure of the designed AlGaN RCE Schottky PD. 


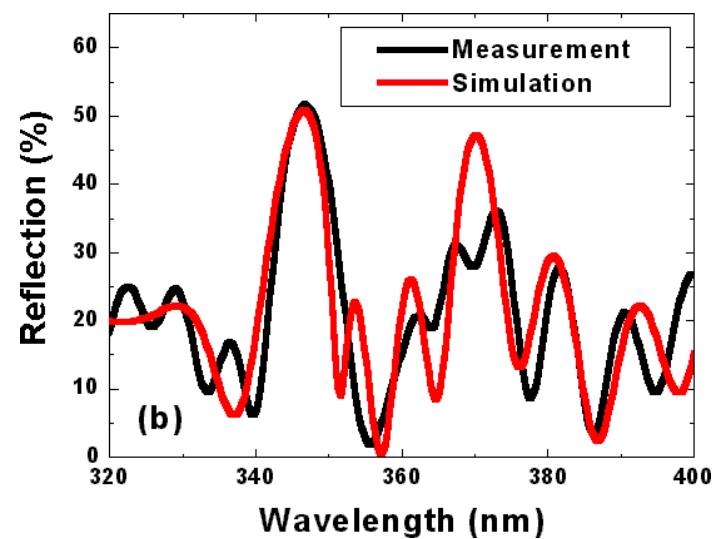

Figure 2. Measured and simulated spectral reflectivity of the RCE-PD wafer.

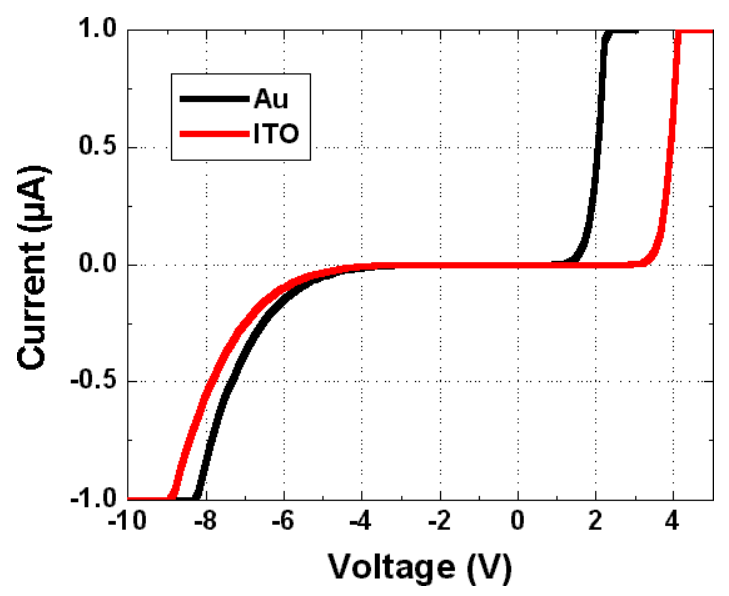

Figure 3. Typical I-V characteristics of $100 \times 100 \mu \mathrm{m} 2 \mathrm{Au}$ and ITO-Schottky RCE-PDs.

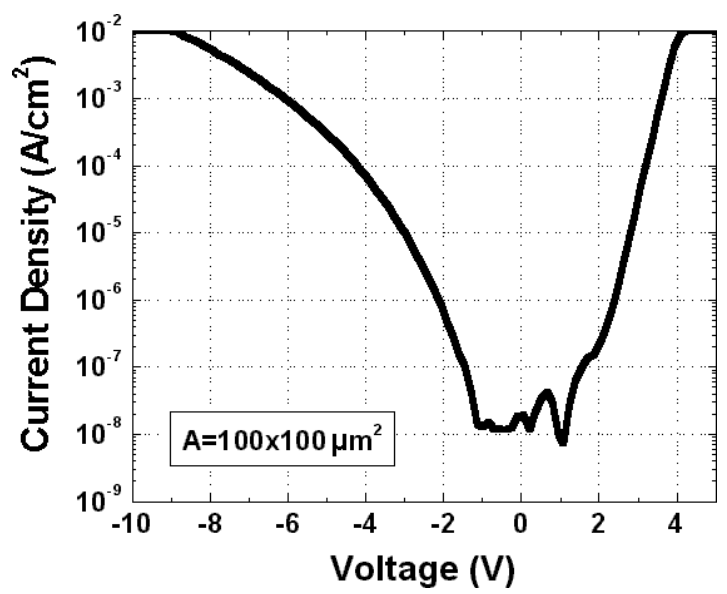

Figure 4. Measured dark current density of a $100 \times 100 \mu \mathrm{m}^{2}$ ITO-Schottky PD.

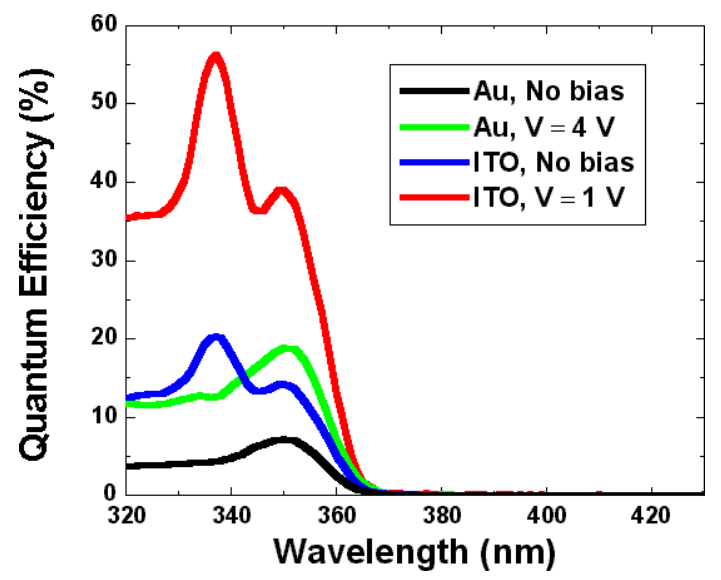

Figure 5. Measured spectral quantum efficiency of Au and ITOSchottky RCE-PDs.

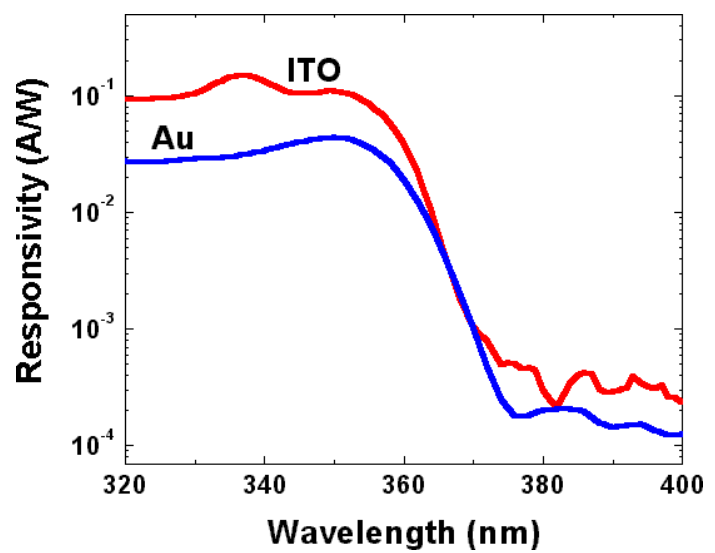

Figure 6. Spectral responsivity curves of biased Au and ITOSchottky RCE-PD samples.

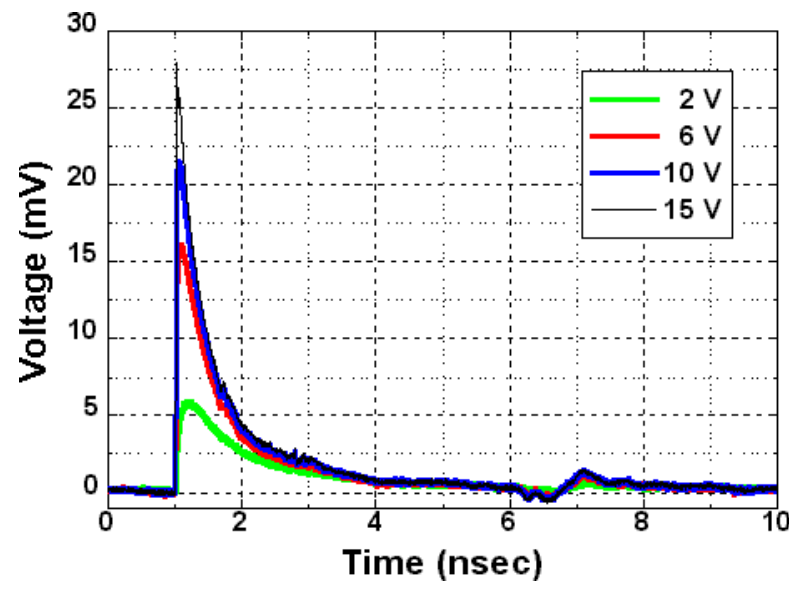

Figure 7. Pulse response of a $150 \times 150 \mu \mathrm{m}^{2} \mathrm{Au}-$ Schottky RCE$\mathrm{PD}$ as a function of reverse bias. 


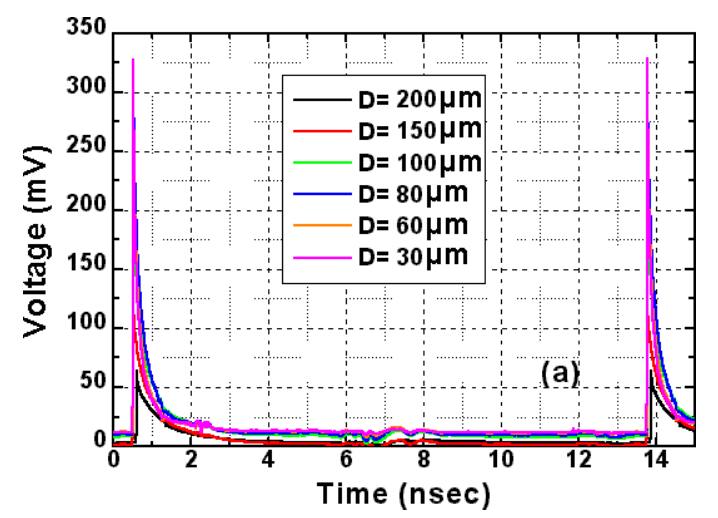

Figure 8a. The area dependence of pulse responses measured at 357 nm. (a) Au-Schottky RCE-PD

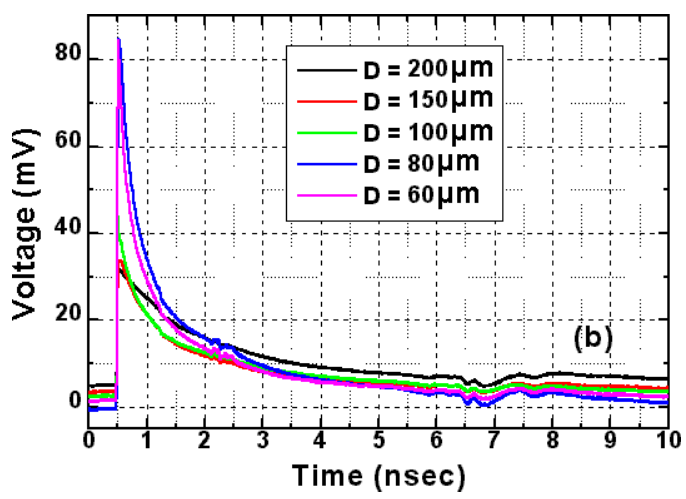

Figure $8 \mathrm{~b}$. The area dependence of pulse responses measured at $357 \mathrm{~nm}$. (b) ITO-Schottky RCE-PD

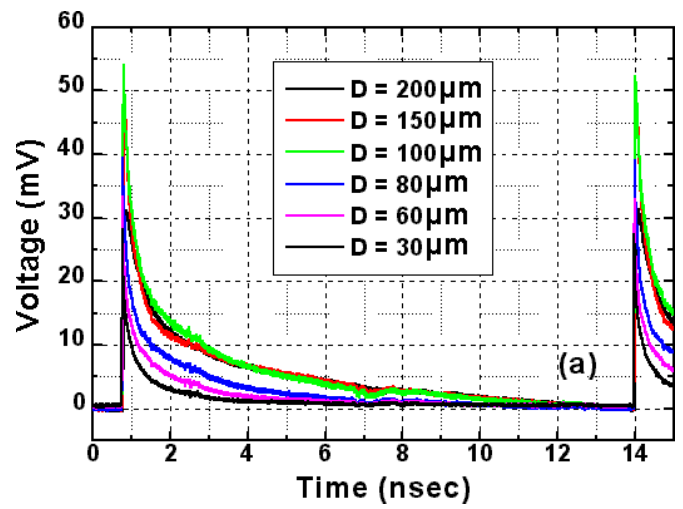

Figure 9a. Temporal pulse responses as a function of device areas measured at $267 \mathrm{~nm}$. (a) Au-Schottky RCE-PD.

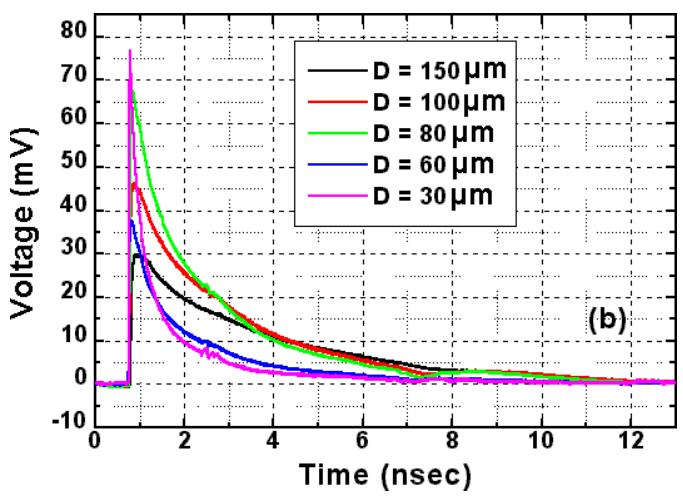

Figure 9b. Temporal pulse responses as a function of device areas measured at $267 \mathrm{~nm}$. (b) ITO-Schottky RCE-PD

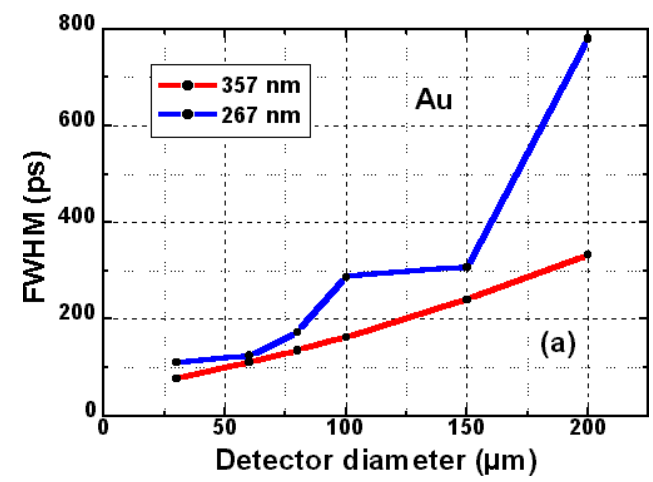

Figure 10a. Measured pulse-width variation with device area under $357 \mathrm{~nm}$ and $267 \mathrm{~nm}$ illumination. (a)Au-Schottky RCEPD .

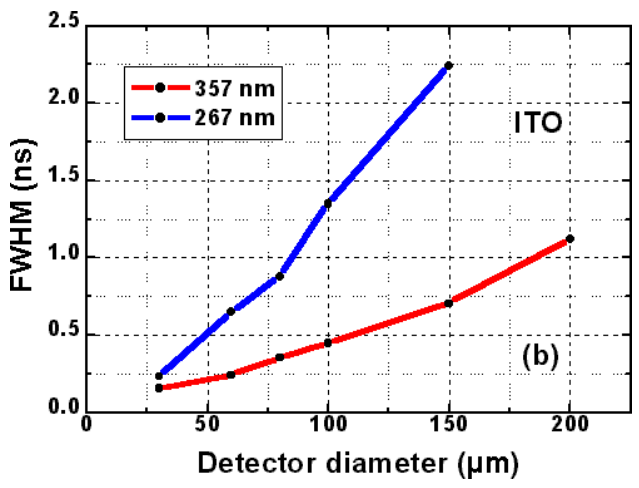

Figure 10b. Measured pulse-width variation with device area under $357 \mathrm{~nm}$ and $267 \mathrm{~nm}$ illumination. (b) ITO-Schottky RCE-PD. 


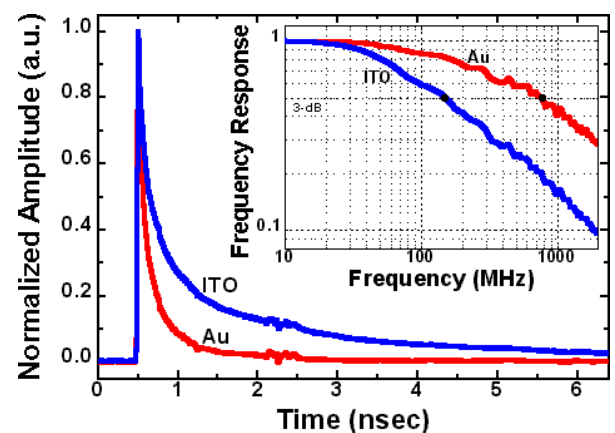

Figure 11. Normalized pulse responses of $30 \mu \mathrm{m}$-diameter $\mathrm{Au}$ and ITO-Schottky RCE-PDs with 77 ps and 154 ps FWHM respectively. Inset shows the corresponding FFT curves. 